Research paper

\title{
The natural history of depression and trajectories of symptoms long term after stroke: The prospective south London stroke register
}

\author{
Salma A. Ayis ${ }^{a, *}$, Luis Ayerbe ${ }^{\mathrm{a}, \mathrm{b}}$, Siobhan L. Crichton ${ }^{\mathrm{a}}$, Anthony G. Rudd ${ }^{\mathrm{a}, \mathrm{c}}$, \\ Charles D.A. Wolfe ${ }^{\mathrm{a}, \mathrm{d}, \mathrm{e}}$ \\ ${ }^{a}$ Division of Health and Social Care Research King's College London, London, UK \\ ${ }^{\mathrm{b}}$ Blizard Institute. Centre for Primary Care and Public Health, Barts and The London School of Medicine and Dentistry, Queen Mary University of London, UK \\ ' Stroke Unit, Guy's and St. Thomas' NS Foundation Trust, St. Thomas' Hospital, London, UK \\ ' National Institute for Health Research (NIHR) Biomedical Research Centre, Guy's and St Thomas' NHS Foundation Trust, London, UK \\ e National Institute for Health Research (NIHR) Collaboration for Leadership in Applied Health Research and Care (CLAHRC) South London at King's College \\ Hospital NHS Foundation Trust, UK
}

\section{A R T I C L E I N F O}

\section{Article history:}

Received 25 October 2015

Received in revised form

15 December 2015

Accepted 12 January 2016

Available online 13 January 2016

Keywords:

Natural history of depression

Depression symptoms

Stroke

Group based trajectory modelling (GBTM)

\begin{abstract}
A B S T R A C T
Background: The natural history of depression in stroke patients is complex and the mechanism of change in symptoms over time is not fully understood. We hypothesise that there are different trajectories of symptoms after stroke.

Methods: The primary analysis comprised 761 patients who completed 5 years follow up, obtained from the prospective South London Stroke Register (1998-2013). The Hospital Anxiety and Depression scale (HADs) was used to screen patients for depression symptoms at 3 months after stroke, then annually. Trajectories of depression symptoms were detected using group based trajectory modelling (GBTM). Results: Four patterns of symptoms (Groups I-IV) were identified: $6.31 \%$ of patients had severe symptoms, improved slightly in early years then worsen (predicted mean HADs score, $15.74(\mathrm{se}=1.06)$ ); $28.65 \%$ had moderate symptoms, a tendency to get worse over time, predicted mean score 7.36 $(\mathrm{se}=0.35) ; 49.54 \%$ had mild symptoms and a tendency of getting worse, predicted mean $3.89(\mathrm{se}=0.30)$, and $15.51 \%$ of the cohort, had no symptoms and remained so over time. The lowest rate of Selective serotonin reuptake inhibitors (SSRI) use, over 5 years after stroke was $1.1 \%$ for group (I) and highest was $35 \%$ for group (IV). Sensitivity analyses were used to assess the robustness of the findings using several inclusion criteria and findings agreed with the primary results.

Limitations: There is loss to follow up of around $20 \%$.

Conclusions: The study identified 4 trajectories of depression symptoms, providing useful information for the long term management of stroke patients and for the implementation of cost effective personalized interventions.
\end{abstract}

(c) 2016 Elsevier B.V. All rights reserved.

\section{Introduction}

Systematic reviews and meta-analysis have recently estimated that depression prevalence at any time point up to 15 years after stroke is around 30\%.(Ayerbe et al., 2013b; Hackett and Pickles, 2014) Depression is associated with disability, poor quality of life, increased mortality, and slow recovery (Lai et al., 2002; Robinson, 2003).

The pattern of depression development over time is not fully understood in stroke patients. Evidence is patchy and

\footnotetext{
* Correspondence to: Department of Primary Care and Public Health Sciences. King's College London, 4th Floor Addison House, Guy's Campus, London SE1 1UL UK.

E-mail address: salma.ayis@kcl.ac.uk (S.A. Ayis).
}

controversial, often suggesting decreasing incidence rates (Aben and Verhey, 2006), increasing (Lincoln et al., 2013) and or dynamic, with episodes of recovery and recurrence over time. (Ayerbe et al., 2013a; Farner et al., 2010; Wade et al., 1987) No study to our knowledge has either formally examined the heterogeneity of developmental patterns over time or used formal diagnostic criteria to estimate the prevalence rates of different severity levels of depression long term after stroke and the association of these with disability and socio-demographic factors.

The hypothesis that a single model or developmental pathway can explain everyone's risk of an outcome or disorder such as depression may be unrealistic. Conventional growth trajectory models use a rather simplified assumption that individuals belong to a single population and estimate a single average trajectory to describe development in the entire population (multilevel random 
effect models (Goldstein and Rasbash, 2003)). Group based trajectory models (GBTM)(Nagin and Odgers, 2010b), and Growth mixture modelling (GMM) (Muthen and Muthen, 2000), are up to date statistical procedures designed to identify clusters of individuals (trajectories) who have followed a similar developmental trajectory of an outcome of interest over time. The methods are increasingly being applied in clinical research and have helped to elucidate important associations, including relationships between different patterns of drug misuse (adolescent-limited versus life course persistent) and the development of antisocial behaviours; the identification of trajectories of prostate specific antigen (PSA) biomarker and the differential development of prostate cancer, have been used to estimate differences in the prevalence of psychiatric disorders among children with and without intellectual disabilities and to identify differential psychosocial exposures for each. (Emerson and Hatton, 2007; Kandel et al., 1992; Moffitt and Klaus-Grawe Think, 2013; Muthen, 2006; Pearson et al., 1994)

In this study we aim: (I) to establish the presence of different patterns of development (trajectories) in depression symptoms long term after stroke, (II) to estimate the prevalence of each, and to examine associations between different patterns and stroke severity, physical disability, and the uptake of antidepressants.

\section{Methods}

\subsection{Design}

Patients were recruited between 1998 and 2013 from the South London Stroke Register (SLSR), a prospective population-based cohort study, and were followed up to June 2014. The World Health Organization (WHO) definition of stroke was used (Hatano, 1976). To increase the completeness of notification sixteen overlapping referral sources were used (Heuschmann et al., 2008). The STROBE flowchart provides details (Fig. 1). Some of the patients who were lost to follow at 3 months were captured at a later time points. Data collected during the acute phase of stroke included socio-demographic factors, medication use, comorbidities and stroke severity, including Glasgow coma scale (GCS) scores (categorized as severe (3-8), moderate (9-12), and mild (13-15) levels of impairment), incontinence, and paresis. Patients were assessed at three months after stroke, one year after stroke and then annually. Follow up at 3 months after stroke was by postal questionnaire or interview. At follow up patients were screened for depression using the Hospital Anxiety and Depression scale (HADs) (Zigmond and Snaith, 1983). HADs comprised 14 items, 7 items screen for depression and 7for anxiety. The scale has been validated in stroke patients and has shown good performance both when it is used by an interviewer and when it is self-administered (Aben et al., 2002). Selective serotonin reuptake inhibitors (SSRI) use pre-stroke, at 3 months, and annually after stroke was reported. As a UK study, we have focused on the use of SSRI only, as these were the most commonly used, well defined as a group, and they cause fewer side effects, according to evidence from the NHS and the National Institute for Health and Care Excellence (NHS, 2015; NICE, 2015).

Patients with impaired communication were not assessed by HADs. Disability was assessed at the acute phase, and at follow up, using the Barthel Index (BI) (Mahoney and Barthel, 1965) categorised as severe disability (0-14); moderate (15-19) and independent (20). The scale was validated for use in stroke patients and was reported to have excellent reliability (Duffy et al., 2013; Wolfe et al., 1991). Primary and sensitivity analyses were performed using different inclusion criteria.

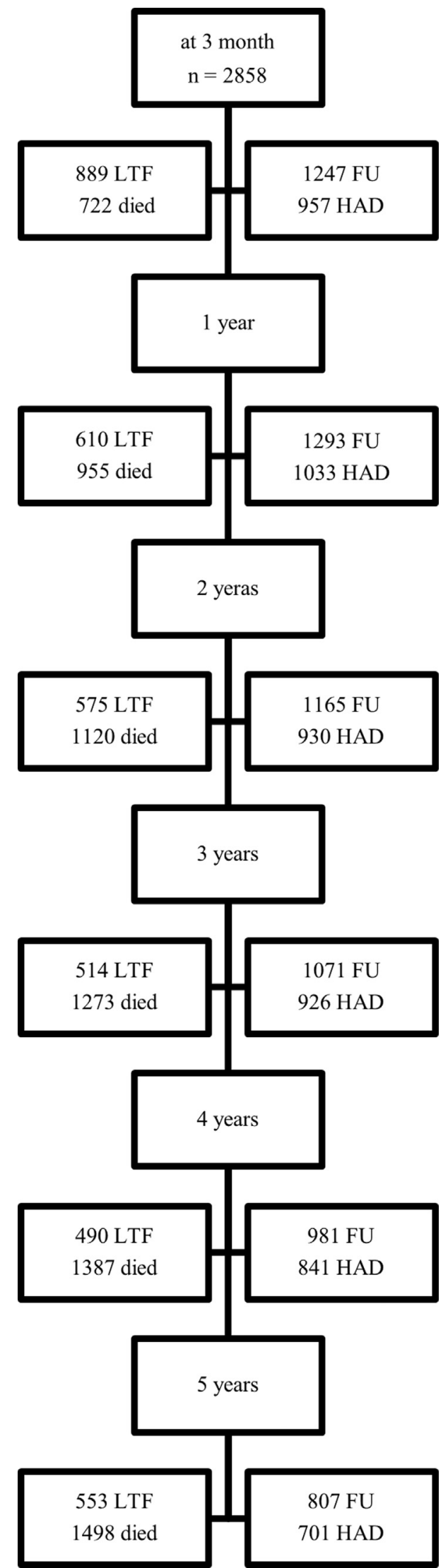

Fig. 1. Flow chart showing the number of stroke patients included at each followup (APA, 2000). [Legend: $n=$ number of patients interviewed; $L T F=$ Lost to follow up; $\mathrm{FU}=$ completed the follow up; $\mathrm{HAD}=\mathrm{HAD}$ completed]. 


\subsection{Primary analysis inclusion criteria}

The primary analysis was confined to patients who had their first-ever stroke before 2009, have complete 5 years of follow up, and have completed the HADs in at least 4 occasions $(n=761)$. The purpose was to ensure a large sample, with reasonably long term follow up, and with good completion rate of HADs.

\subsection{Sensitivity analyses inclusion criteria}

The criteria used were: (a) patients with 4 or more measurements of HADs between 3 months to 5 years after stroke, $(n=852)$; (b) patients with 7or more measurement of HADs between 3 months to 10 years after stroke $(n=613)$; (c) patients with 8 or more measurements of HADs between 3 months to 15 years after stroke $(n=350)$, and (d) patients with 4 or more measurements of HADs during the 15 years follow up $(n=1061)$.

These analyses were performed for two reasons: (1) to assess the robustness of the results from the primary analysis using different lengths of follow up times and different numbers of observations per participant, and (2) to examine the impact of important time varying and time invariant covariates including anxiety, the use of antidepressants, gender and age at stroke onset, on the shapes and the membership of the trajectories obtained by the primary analysis.

\subsection{Statistical analysis}

The study conformed to the Strengthening the Reporting of Observational Studies in Epidemiology (STROBE) recommendations (von Elm et al., 2007). Chi squared test was used to compare proportions for categorical variables and the Analysis of Variance (ANOVA) and Kruskal-Wallis were used for continuous variables. $P$ value $\leq 0.05$ was used as a criterion for significance.

The probability that a subject belongs to a latent class (trajectory), with each class representing a sub-population was modelled using the censored normal (Tobit) model. The model was recommended for the analysis of repeated measurements of continuous scores such as HADs' (Nagin and Odgers, 2010a). HADs depression scores over time were used as continuous outcome. The software developed by Jones and Nagin, for the analysis of GBTM was installed in Stata and the command "traj" was used (Jones, 2013). The model has the capability to adjust for the effect of time varying or time invariant covariates on the probability of group membership and on the shape of the trajectories (Nagin, 2005). We used these features in a sensitivity analysis to examine the effect of time varying factors: anxiety and antidepressant use, and the time invariant: gender, and age at stroke onset, on the shape of development over time and on the patients' membership to trajectories.

A range of (1-8) trajectories were examined, and shapes that range between 1 st and 3 rd order polynomial functions of time since stroke for each trajectory, were specified and tested. Three different considerations were used to decide on the number and shapes of the selected trajectories: (1) the Bayesian information Criterion (BIC) statistics, a low BIC indicates well-fitting model; (2) the classification quality using the posterior probabilities: the average posterior probability for the assignment of individuals to a class should be considerably higher than the average posterior probabilities for the assignment of these individuals to other classes, and (3) the meaning of the classification, judging the similarity and differences between trajectories, the size of each, and the usefulness in practice. (Muthen and Muthen, 2000; Nagin and Odgers, 2010b)

The socio-demographic characteristics, stroke severity and antidepressants use for the final groups defined by the primary analysis were estimated. Groups were compared in selected characteristics only, for example, the proportion with severe physical function limitations, due to the large number of possible comparisons, and the resulting inflation of Type I error. The software Stata (Version 13) (StataCorp., (2013). Stata Statistical Software: Release 13. College Station) was used for all analyses.

\section{Results}

\subsection{Summary of main features of the register data}

The STROBE flowchart provides details of patients recruited between 1998 and 2013 follow up data (Fig. 1). Appendix 1 (Supplementary), provides a summary comparison of the demography and stroke severity of patients who died, lost to follow at 3 months, and those who completed the 3 month's assessments. Briefly, patients who died have more severe stroke, and were about 10 years older (mean age 74.8; $\mathrm{SD}=14.5$ ) than those followed, or lost to follow. Patients who were lost to follow, are slightly younger than those who were followed, mean age (66.29; $\mathrm{SD}=15.89$ ) and (69.59; $\mathrm{SD}=13.63)$, for the two groups respectively. Comparisons with respect to other factors did not show appreciable differences. It is however, inappropriate to make conclusions about differences, due to the large proportions of missing data in the patients who were lost to follow.

\subsection{Primary analysis results}

The primary analysis comprised 761 patients, identified 4 developmental trajectories of depression symptoms. Estimates of the parameters of these were displayed in Table 1 . The first trajectory (Group I) represents patients with no depression symptoms (15.51\%) who remain so over time (polynomial type: constant); Group II, includes patients with mild symptoms (49.54\%), and a tendency for a slight increase in symptoms over time (polynomial type: positive linear); Group III, has patients with moderate symptoms (28.65\%), and a stronger tendency for deterioration over time (polynomial type: positive linear), and Group IV has patients with severe symptoms (6.31\%), a tendency for a significant decrease, followed by an increase (polynomial type: linear and quadratic).

The censored Tobit model fits the data well and the four trajectories' solution was taken as best solution using the criteria described in methods, including the Bayesian information criterion (BIC), Akaike information criterion (AIC), group membership

Table 1

Estimates of 4 trajectories of depression from 3 months to 5 years after stroke.

\begin{tabular}{llclc}
\hline Group & Parameter & Estimate & Standard Error & $P$ value \\
\hline I & Intercept & 1.18 & 0.38 & $<0.001$ \\
& Intercept & 3.89 & 0.30 & $<0.001$ \\
II & Linear & 0.14 & 0.05 & 0.01 \\
III & Intercept & 7.36 & 0.35 & $<0.001$ \\
& Linear & 0.24 & 0.07 & $<0.001$ \\
IV & Intercept & 15.74 & 1.06 & $<0.001$ \\
& Linear & -1.79 & 0.67 & 0.01 \\
& Quadratic & 0.24 & 0.09 & 0.01 \\
& n & $\%$ & $(95 \% \mathrm{CI})$ & $18.26 \%$ \\
I & 118 & $15.51 \%$ & $13.10 \%$ & $53.10 \%$ \\
II & 377 & $49.54 \%$ & $45.99 \%$ & $31.97 \%$ \\
III & 218 & $28.65 \%$ & $25.54 \%$ & $8.28 \%$ \\
IV & 48 & $6.31 \%$ & $4.78 \%$ & \\
Total & 761 & & & \\
\hline
\end{tabular}

Note. Group I-IV stand for patients within the 4 trajectories of depression identified in the primary analysis. 
Table 2

Mean posterior probabilities of assignment of groups (I-IV) for members of each group

\begin{tabular}{lllll}
\hline & Mean & SD & Min & Max \\
\hline Group & & & & \\
I & $\mathbf{0 . 8 3 2}$ & 0.152 & 0.500 & 0.999 \\
II & 0.086 & 0.121 & 0.000 & 0.489 \\
III & 0.000 & 0.000 & 0.000 & 0.000 \\
IV & 0.000 & 0.000 & 0.000 & 0.000 \\
Group II & & & & \\
I & 0.168 & 0.152 & 0.000 & 0.500 \\
II & $\mathbf{0 . 8 3 6}$ & 0.128 & 0.502 & 0.985 \\
III & 0.093 & 0.128 & 0.000 & 0.496 \\
IV & 0.000 & 0.000 & 0.000 & 0.000 \\
Group III & & & & \\
I & 0.000 & 0.000 & 0.000 & 0.000 \\
II & 0.078 & 0.121 & 0.000 & 0.497 \\
III & $\mathbf{0 . 8 7 3}$ & 0.138 & 0.502 & 0.998 \\
IV & 0.062 & 0.114 & 0.000 & 0.495 \\
Group IV & & & & \\
I & 0.000 & 0.000 & 0.000 & 0.000 \\
II & 0.000 & 0.000 & 0.000 & 0.000 \\
III & 0.034 & 0.094 & 0.00 & 0.497 \\
IV & $\mathbf{0 . 9 3 8}$ & 0.114 & 0.505 & 0.999 \\
\hline
\end{tabular}

Note. SD: Standard deviation. 0.000 indicates very small values, approximated to the nearest 3 decimal places.

probabilities, and the meaningful and sizes of the groups. Although BIC was lowest for a 5 groups' solution, the addition of a 5 th group only decreases the BIC slightly, and created an intermediate group between groups I and II, including patients with mild symptoms, and with characteristics between those for groups I and II which was not considered a meaningful addition. The BIC and AIC followed a fairly similar patterns over the 8 models examined. The BIC and AIC corresponding to1-8 trajectories examined were given by supplementary Fig. S1.

The average posterior probabilities of assignment to groups were very high for members assigned to each group, compared to the probabilities of these being assigned to other groups (Table 2). The highest average posterior probability was 0.938 , for the assignment of members to Group IV, and the lowest was 0.832 (considered fairly high) was the average probability for the assignment of members to Group I.

Table 3, summarizes the demography, and measures of stroke severity in the acute phase, including paresis, GCS, incontinence, and physical function for the four groups. The table shows that Group I, has younger patients than Groups II and III, has marked majority of males (66.10\%), less severe stroke, and the lowest proportion of severe physical function limitations (17.80\%) in the acute phase, compared to $26.3 \%, 33.9 \%$ and $35.4 \%$, for the groups II-IV respectively. The group with severe depression symptoms (Group IV) has higher rates of impairments, paresis, and physical function limitations, compared to the other groups. The two intermediate groups (II and III) have significantly higher proportions of males, and of older patients compared to Group IV. The ethnic structure also differs across groups, notably Groups II and III have higher proportions of Blacks compared to others.

The physical function status and use of SSRI, between 3 months and 5 years after stroke for the 4 groups (I-IV) are presented in Table 4 . Throughout the 5 years of follow up, Group I, and Group II, showed consistently better physical function than the other groups. Differences across groups were significant ( $p$ value $<0.001$ ). Group I, has over $70 \%$ of fully independent patients up to 3 years, and over $60 \%$ of those, up to 5 years after stroke. The proportions of fully independent patients decreased over time and were remarkably small for Group IV. Antidepressants' (SSRI) use pre stroke was low in all groups, and the difference was not significant at the $5 \%$ level. Post stroke use differ across groups
Table 3

Demography and stroke severity in the acute phase for four groups of patients.

\begin{tabular}{|c|c|c|c|c|c|}
\hline Group & I & II & III & IV & Total \\
\hline Group size $(n)$ & 118 & 377 & 218 & 48 & 761 \\
\hline Mean age & 62.02 & 66.20 & 64.38 & 60.59 & 64.67 \\
\hline (SD) & 13.36 & 13.09 & 12.96 & 10.75 & (13.06) \\
\hline \multicolumn{6}{|l|}{ Sex } \\
\hline Male & $66.10 \%$ & $55.40 \%$ & $54.10 \%$ & $47.90 \%$ & $56.20 \%$ \\
\hline Female & $33.90 \%$ & $44.60 \%$ & $45.90 \%$ & $52.10 \%$ & $43.80 \%$ \\
\hline \multicolumn{6}{|l|}{ Ethnicity } \\
\hline White & $72.00 \%$ & $68.20 \%$ & $66.10 \%$ & $72.90 \%$ & $68.50 \%$ \\
\hline Black & $22.00 \%$ & $24.70 \%$ & $29.40 \%$ & $12.50 \%$ & $24.80 \%$ \\
\hline Others & $4.20 \%$ & $5.60 \%$ & $3.70 \%$ & $14.60 \%$ & $5.40 \%$ \\
\hline Unknown & $1.70 \%$ & $1.60 \%$ & $0.90 \%$ & $0.00 \%$ & $1.30 \%$ \\
\hline \multicolumn{6}{|l|}{ Paresis } \\
\hline No & $50.00 \%$ & $30.20 \%$ & $17.00 \%$ & $20.80 \%$ & $28.90 \%$ \\
\hline Yes & $39.80 \%$ & $57.60 \%$ & $67.00 \%$ & $62.50 \%$ & $57.80 \%$ \\
\hline Unknown & $0.80 \%$ & $0.80 \%$ & $0.90 \%$ & $0.00 \%$ & $0.80 \%$ \\
\hline \multicolumn{6}{|l|}{ Incontinence } \\
\hline No & $89.80 \%$ & $78.50 \%$ & $71.60 \%$ & $81.30 \%$ & $78.40 \%$ \\
\hline Yes & $6.80 \%$ & $19.40 \%$ & $24.80 \%$ & $14.60 \%$ & $18.70 \%$ \\
\hline Unknown & $3.40 \%$ & $2.10 \%$ & $3.70 \%$ & $4.20 \%$ & $2.90 \%$ \\
\hline \multicolumn{6}{|l|}{ Glasgow Coma Scale (GCS) } \\
\hline Severe & $2.70 \%$ & $1.60 \%$ & $3.30 \%$ & $2.10 \%$ & $2.30 \%$ \\
\hline Moderate & $2.70 \%$ & $6.50 \%$ & $8.60 \%$ & $4.30 \%$ & $6.40 \%$ \\
\hline Mild & $94.70 \%$ & $91.80 \%$ & $88.10 \%$ & $93.60 \%$ & $91.30 \%$ \\
\hline \multicolumn{6}{|c|}{ Barthel Index (BI) at the cute phase } \\
\hline Severe limitations (0-14) & $17.80 \%$ & $26.30 \%$ & $33.90 \%$ & $35.40 \%$ & $27.70 \%$ \\
\hline Moderate limitations (15-19) & $12.70 \%$ & $18.60 \%$ & $18.80 \%$ & $20.80 \%$ & $17.90 \%$ \\
\hline Independent (20) & $55.90 \%$ & $36.10 \%$ & $27.50 \%$ & $27.10 \%$ & $36.10 \%$ \\
\hline Unknown & $13.60 \%$ & $19.10 \%$ & $19.70 \%$ & $16.70 \%$ & $18.30 \%$ \\
\hline
\end{tabular}

Note. Group I-IV stand for patients within the 4 trajectories of depression identified in the primary analysis.

throughout the 5 years, and the differences were significant ( $p$ value $<0.001$ ). SSRI use was consistent with depression symptoms, with lowest rates of use being observed in group I, and highest rates in group IV, with highest proportion observed being $35 \%$.

The 4 trajectories of the primary analysis were graphically presented along with the observed mean HADs' scores, at each follow up time in Fig. 2. No differences were noted between the observed means and medians of the scores over the 5 years follow up. The means were presented to illustrate the agreement between the observed and predicted means, as the Tobit model is based on prediction of means. The observed means in panel (a) and the predicted means in panel (b) were in good agreement over the 5 years and for the 4 trajectories (I-IV).

\subsection{Sensitivity analyses results}

The estimates of the 4 trajectories based on inclusion criteria (a) and (b), were presented in Appendix 2, and those based on inclusion criteria (c) and (d), in Appendix 3. The numbers and shapes of the primary analysis trajectories were maintained for all data sets. For (d) however, the quadratic term was not significant in the sensitivity analysis; removing the quadratic term, did not alter groups' membership.

The agreements between the groups derived from the primary analysis using a strict criterion and those based on other larger and smaller samples using different criteria (a-d) were high. Kappa values range between $0.67(\mathrm{se}=0.04)$ and $0.97(\mathrm{se}=0.02)$ and weighted Kappa between $0.86(\mathrm{se}=0.04)$ and $0.99(\mathrm{se}=0.04)$. Adjustment for the covariates did not alter the significance of any of the shape parameters, and the effect on membership probabilities was negligible (not presented). 
Table 4

Physical limitations and antidepressants (SSRI) use before and 5 years after stroke for groups (I-IV) of patients.

\begin{tabular}{|c|c|c|c|c|c|}
\hline Group & $\mathbf{I}$ & II & III & IV & Total \\
\hline Size $(n)$ & 118 & 377 & 218 & 48 & 761 \\
\hline \multicolumn{6}{|l|}{ BI at 3 months after stroke } \\
\hline Severe limitations (0-14) & $0.00 \%$ & $6.10 \%$ & $18.80 \%$ & $29.20 \%$ & $10.20 \%$ \\
\hline Moderate limitations (15-19) & $15.30 \%$ & $27.60 \%$ & $36.70 \%$ & $31.30 \%$ & $28.50 \%$ \\
\hline Independent (20) & $60.20 \%$ & $41.10 \%$ & $22.00 \%$ & $14.60 \%$ & $36.90 \%$ \\
\hline Unknown & $24.60 \%$ & $25.20 \%$ & $22.50 \%$ & $25.00 \%$ & $24.30 \%$ \\
\hline \multicolumn{6}{|l|}{ BI at 1 Year } \\
\hline Severe limitations $(0-14)$ & $0.00 \%$ & $5.60 \%$ & $19.30 \%$ & $25.00 \%$ & $9.90 \%$ \\
\hline Moderate limitations (15-19) & $14.40 \%$ & $36.60 \%$ & $37.60 \%$ & $31.30 \%$ & $33.10 \%$ \\
\hline Independent (20) & $78.00 \%$ & $48.80 \%$ & $33.50 \%$ & $25.00 \%$ & $47.40 \%$ \\
\hline Unknown & $7.60 \%$ & $9.00 \%$ & $9.60 \%$ & $18.80 \%$ & $9.60 \%$ \\
\hline \multicolumn{6}{|l|}{ BI at 2 Years } \\
\hline Severe limitations (0-14) & $0.00 \%$ & $8.20 \%$ & $18.30 \%$ & $16.70 \%$ & $10.40 \%$ \\
\hline Moderate limitations (15-19) & $20.30 \%$ & $35.00 \%$ & $41.30 \%$ & $56.30 \%$ & $35.90 \%$ \\
\hline Independent (20) & $71.20 \%$ & $48.30 \%$ & $27.10 \%$ & $10.40 \%$ & $43.40 \%$ \\
\hline Unknown & $8.50 \%$ & $8.50 \%$ & $13.30 \%$ & $16.70 \%$ & $10.40 \%$ \\
\hline \multicolumn{6}{|l|}{ BI at 3Years } \\
\hline Severe limitations (0-14) & $1.70 \%$ & $9.00 \%$ & $19.70 \%$ & $33.30 \%$ & $12.50 \%$ \\
\hline Moderate limitations (15-19) & $13.60 \%$ & $35.50 \%$ & $44.50 \%$ & $39.60 \%$ & $35.00 \%$ \\
\hline Independent (20) & $74.60 \%$ & $46.90 \%$ & $24.80 \%$ & $14.60 \%$ & $42.80 \%$ \\
\hline Unknown & $10.20 \%$ & $8.50 \%$ & $11.00 \%$ & $12.50 \%$ & $9.70 \%$ \\
\hline \multicolumn{6}{|l|}{ BI at 4 Years } \\
\hline Severe limitations (0-14) & $0.80 \%$ & $7.40 \%$ & $27.50 \%$ & $29.20 \%$ & $13.50 \%$ \\
\hline Moderate limitations (15-19) & $17.80 \%$ & $38.50 \%$ & $39.90 \%$ & $35.40 \%$ & $35.50 \%$ \\
\hline Independent (20) & $66.10 \%$ & $41.40 \%$ & $19.70 \%$ & $14.60 \%$ & $37.30 \%$ \\
\hline Unknown & $15.30 \%$ & $12.70 \%$ & $12.80 \%$ & $20.80 \%$ & $13.70 \%$ \\
\hline \multicolumn{6}{|l|}{ BI at 5 Years } \\
\hline Severe limitations (0-14) & $1.70 \%$ & $9.80 \%$ & $23.40 \%$ & $33.30 \%$ & $13.90 \%$ \\
\hline Moderate limitations (15-19) & $14.40 \%$ & $31.30 \%$ & $31.20 \%$ & $25.00 \%$ & $28.30 \%$ \\
\hline Independent (20) & $62.70 \%$ & $32.40 \%$ & $15.60 \%$ & $14.60 \%$ & $31.10 \%$ \\
\hline Unknown & $21.20 \%$ & $26.50 \%$ & $29.80 \%$ & $27.10 \%$ & $26.70 \%$ \\
\hline \multicolumn{6}{|l|}{ Antidepressants (SSRI) use } \\
\hline Pre stroke & $0.80 \%$ & $2.70 \%$ & $3.70 \%$ & $6.30 \%$ & $2.90 \%$ \\
\hline 3 months after stroke & $1.10 \%$ & $4.20 \%$ & $16.00 \%$ & $23.70 \%$ & $8.40 \%$ \\
\hline 1 year & $3.70 \%$ & $5.20 \%$ & $12.40 \%$ & $32.50 \%$ & $8.60 \%$ \\
\hline 2 years & $1.80 \%$ & $4.60 \%$ & $16.80 \%$ & $35.00 \%$ & $9.30 \%$ \\
\hline 3 years & $1.80 \%$ & $5.90 \%$ & $19.40 \%$ & $26.70 \%$ & $10.40 \%$ \\
\hline 4 years & $2.80 \%$ & $6.70 \%$ & $18.40 \%$ & $21.40 \%$ & $10.40 \%$ \\
\hline 5 years after stroke & $2.00 \%$ & $7.50 \%$ & $20.70 \%$ & $24.30 \%$ & $11.40 \%$ \\
\hline
\end{tabular}

Note. SSRI stands for 5 selective serotonin reuptake inhibitors; BI: Barthel Index. Overall ( $P$ values) for all comparisons across groups were $<0.001$, except $p$-value for the comparison of pre stroke SSRI use $(P$ value $=0.86)$.

\section{Discussion}

The study identified 4 groups of stroke patients with remarkable differences in the mechanisms of the development of depression symptoms over time providing further insight into the natural history of depression after stroke. The groups include patients with severe symptoms (6.31\%) that may decrease slightly at early years before getting worse again, moderate $(28.65 \%)$ and mild symptoms (49.54\%) that increase over time, and patients who were free of depression symptoms (15.51\%), and remain so over time.

Estimates on the prevalence of depression after stroke, varied considerably across studies. A pooled estimate of 31\% (95\% CI: $28 \%$ to $35 \%$ ) in stroke survivors at any time up to 5 years after stroke, has been reported in a recent systematic review including 61 studies (Hackett and Pickles, 2014). Our findings supplement current knowledge on overall prevalence, by providing details on different levels of symptoms and varying patterns of development over time. Awareness of the presence of such a range of prognosis with different needs represents additional and timely evidence that is currently needed for the effective and cost effective applications of stratified medicine. It may also be useful for the appropriate assignment of patients to interventions in clinical trials.

Researchers and clinicians have widely acknowledged that depression needs clinical attention in the long term but the poor prognosis of vulnerable patients makes long term interventions difficult to develop and to deliver (McKevitt et al., 2011; Wolfe et al., 2011). A better understanding of the long term development is necessary for the implementation of effective strategies for rehabilitation and long term management. Our results suggest the need for clinicians to consider differences in the development of depression symptoms in stroke patients, acknowledging that patients with moderate and severe symptoms are at high risk for remaining depressed or even getting worse. The significant risk of increase in mild symptoms noted soon after stroke in $49.54 \%$ of patients, also suggest the need for periodic clinical attention in the long term to be considered for appropriate management.

While 5 years follow up was chosen for the primary analysis as short enough for a large number of patients to be still alive and to contribute data at several occasions, the sensitivity analyses have covered 10 , and 15 years of follow up, and the 4 group structure remained.

The use of GMM and GBTM methods is increasing and recent applications have strengthened the evidence on their value. Through better understanding of patients' heterogeneity the methods allowed the discovery of differential patterns in the progress of biomarkers, behavioural disorders, response to interventions, and recovery patterns (Brown et al., 2008; Haviland et al., 2007; Lin et al., 2000; Peer and Spaulding, 2007).

To our knowledge no large scale quantitative study has attempted to describe trajectories of depression symptoms after stroke. A qualitative study however, has closely followed up 23 stroke patients for 12 months after stroke and has identified 4 trajectories of psychological distress (White et al., 2012). Although the two studies differ in methodology, follow up time, scales used, the focus being on disturbed mood rather than depression symptoms in the earlier study, but nonetheless, there are similarities in the developmental features of symptoms that are highly related, in the two studies, and in particular for two groups. The first group, defined as "Resilient", where patients remain in a stable good mood throughout 12 months follow up, resembles, Group I, identified in our study. This may be interpreted that some patients continue to have no depression symptoms long term after stroke. In addition our study has shown that majority of this group were males, fully independent and have maintained physical function independence long term after stroke. The second group, described as "on going mood disturbance trajectory" comprising patients with ongoing feelings of difficulty that continued and exacerbated over time, bears fair similarity to Groups III and IV defined in our study, suggesting that features of severe psychological problems reported for 5 patients over 12 months after stroke, may persist 5 years or longer and the prevalence of these is high (over $30 \%$ ) in stroke patients.

Researchers have reported contradicting associations with antidepressants' use, including positive ones such as improved physical function, and negative such as the increased risk of stroke and mortality, and have questioned the long term safety, the clarity and availability of guidelines for their optimal use (Ayerbe et al., 2014; Coupland et al., 2011; Hackett et al., 2008; Rayner et al., 2011). Our study has shown that these medications were used by between, $21 \%$ and $35 \%$ of patients with severe symptoms over 5 years after stroke, and rates decreases consistently with milder symptoms. For appropriate interpretation of these estimates however, data on compliance, safety, and guidelines for long term use that were reportedly patchy or unavailable would be necessary.

The strengths of this study included the use of long term follow up data derived from an established, prospective, population based stroke register, with $88 \%$ of incidents stoke occurring in the study area being registered, as reported by a capture recapture analysis (Tilling et al., 2001); a completion rate of HADs of over 


\section{Observed and Predicted Mean Depression Scores In Four Trajectories of Depression 5 Years after Stroke}

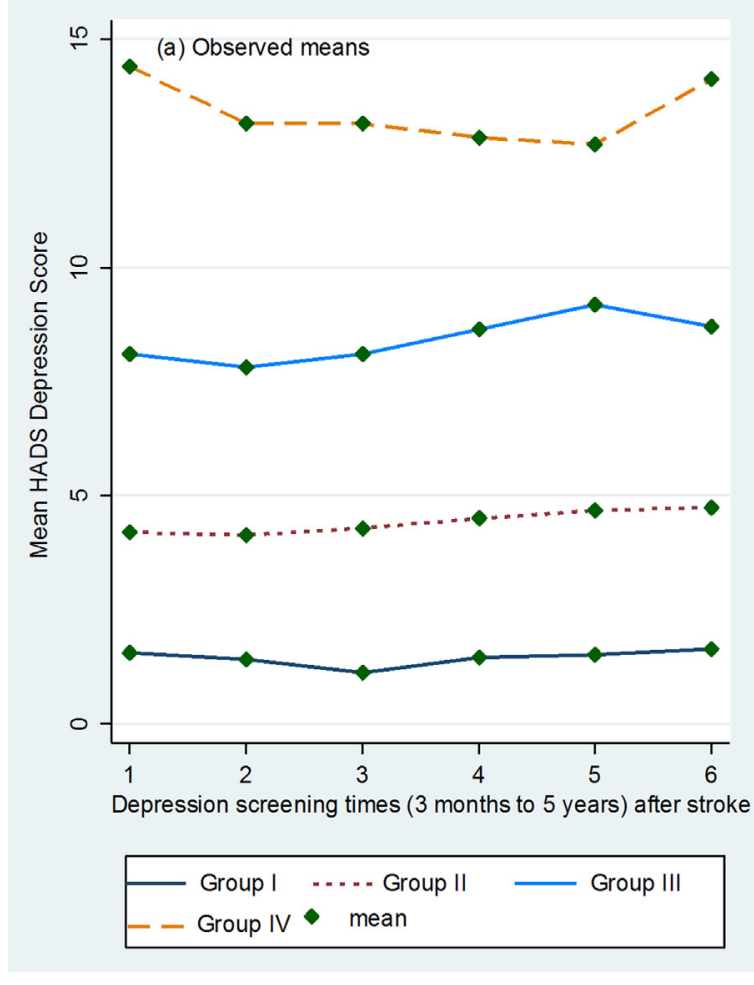

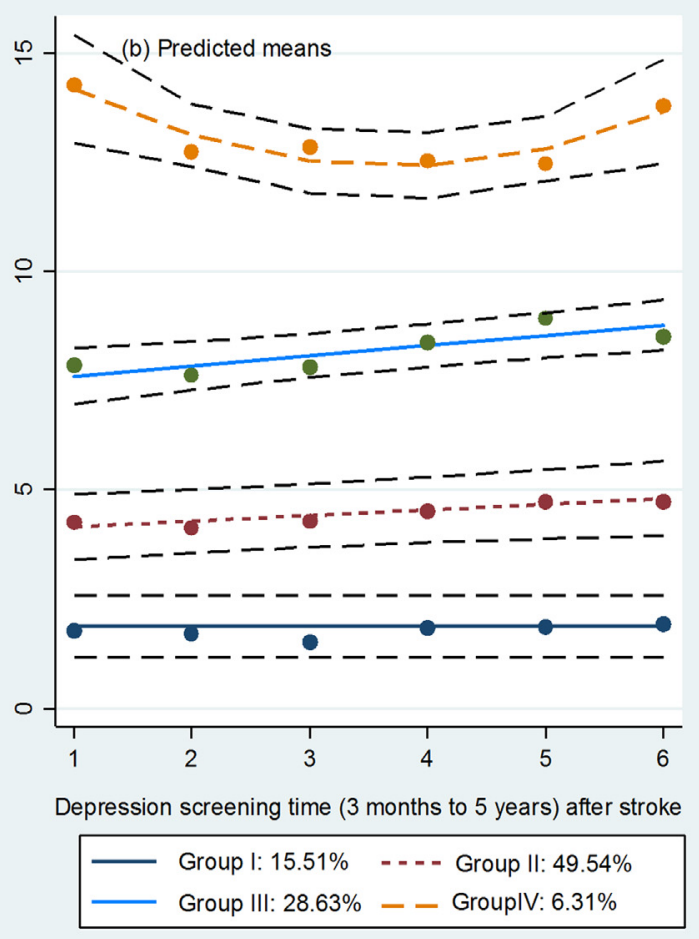

Fig. 2

$75 \%$, and up to $87 \%$; the use of GBTM to define subgroups of different developmental patterns of depression based on formal statistical methodology that provide the necessary tools to distinguish real from random variations in behaviour, such distinction is not possible using ad-hoc classification; the models allow the recognition of the HADs scores as continuous measures overcoming the difficulty of choice of best threshold, as there is no full agreement across studies on that and findings from systematic reviews have shown a wide range (4-11) of cut off points being used.

On the limitation side however, there is loss to follow up of around $20 \%$ on average, and women have slightly higher rates of loss to follow up compared to men. Part of the missing data were due to the inability of some patients with cognitive or communication impairment to respond to the HADs. While this may introduce bias, estimates in the main baseline characteristics from patients with complete data showed no significant differences from those lost to follow up. A method's related limitation is that the existence of sub-groups must be assumed a priori (Nagin and Odgers, 2010b). Assessing depression with a screening scale may represent a limitation, however, although HADs was originally developed as a screening tool for anxiety and depression in hospital patients, it has been validated for use in stroke patients and in the general population (Aben et al., 2002). A recent meta-analysis has shown an optimum performance of HADS' depression, for identifying any depression with 0.85 (95\% CI: 0.78-0.91) sensitivity, and 0.69 (95\% CI: 0.60-0.76) specificity, and for identifying severe depression with 0.82 (95\% CI: $0.70-0.90)$ sensitivity, and 0.76 (95\% CI: 0.72-0.80) specificity (Meader et al., 2014) where the reference standard was diagnosis according to DSM or ICD criteria (World Health Organization., 1993; APA, 2000).

Finally despite the good agreement in the number and shapes of trajectories based on different assumptions, and different samples internally using sensitivity analysis, there remains the need to externally validate these trajectories using independent data sets.

In summary, this study has identified for the first time 4 subgroups of patients with varying levels of depression symptoms and with distinct mechanisms of change in symptoms over time, furthering knowledge for better understanding of the natural history of depression after stroke. The study estimated the prevalence of different levels of severity in depression symptoms and the rates of antidepressants use by each. Awareness of patients with different prognosis represents an additional knowledge that is useful for the implementation of personalized interventions and for the appropriate stratification of patients in clinical trials.

\section{Authors' contributions}

C.D.A.W. and A.G.R. secured the funding. All authors have contributed to the intellectual content of the study. L.A. collected part of the data. SA conducted the statistical analysis and wrote the first draft of the manuscript. All authors contributed to the writing and interpretation of data. All authors have critically reviewed the paper at all stages, and have approved the final copy.

\section{Ethics}

Patients or their relatives gave written informed consent to the SLSR field workers at the time of registration. The ethics committees of Guy's and St. Thomas' Hospital National Health Service Foundation Trust, King's College Hospital Foundation, National Hospital for Nervous Diseases, Queen's Square Hospital, St. George's Hospital, and Westminster Hospital approved the study. 
Conflict of interest

None

\section{Financial support}

The study was funded by Guy's and St Thomas' Hospital Charity, The Stroke Association, Department of Health HQIP Grant, UK, National Institute for Health Research Programme Grant (RP-PG0407-10184). The authors (CW) acknowledge financial support from the Department of Health via the National Institute for Health Research (NIHR) Biomedical Research Centre award to Guy's \& St Thomas' NHS Foundation Trust in partnership with King's College London. CDAW is an NIHR Senior Investigator. This article presents independent research commissioned by the National Institute for Health Research (NIHR) under its Programme Grants for Applied Research funding scheme (RP-PG-0407-10184).

"The research was also supported by the National Institute for Health Research (NIHR) Collaboration for Leadership in Applied Health Research and Care South London at King's College Hospital NHS Foundation Trust. The views expressed are those of the author(s) and not necessarily those of the NHS, the NIHR or the Department of Health."

\section{Acknowledgements}

None.

\section{Appendix A. Supplementary material}

Supplementary data associated with this article can be found in the online version at http://dx.doi.org/10.1016/j.jad.2016.01.030.

\section{References}

Aben, I., Verhey, F., 2006. Depression after a cerebrovascular accident. The importance of the integration of neurobiological and psychosocial pathogenic models. Panminerva Med. 48, 49-57.

Aben, I., Verhey, F., Lousberg, R., Lodder, J., Honig, A., 2002. Validity of the beck depression inventory, hospital anxiety and depression scale, SCL-90, and hamilton depression rating scale as screening instruments for depression in stroke patients. Psychosomatics 43, 386-393.

APA, 2000. Diagnostic and statistical manual of mental disorders: DSM-IV-TR, 4th ed. American Psychiatric Association, Washington, DC.

Ayerbe, L., Ayis, S., Crichton, S., Wolfe, C.D., Rudd, A.G., 2013a. The natural history of depression up to 15 years after stroke: the South London stroke register. Stroke 44, 1105-1110.

Ayerbe, L., Ayis, S., Crichton, S.L., Rudd, A.G., Wolfe, C.D., 2014. Explanatory factors for the increased mortality of stroke patients with depression. Neurology 83, 2007-2012.

Ayerbe, L., Ayis, S., Wolfe, C.D., Rudd, A.G., 2013b. Natural history, predictors and outcomes of depression after stroke: systematic review and meta-analysis. Br. J. Psychiatry 202, 14-21.

Brown, C.H., Wang, W., Kellam, S.G., et al., 2008. Methods for testing theory and evaluating impact in randomized field trials: intent-to-treat analyses for integrating the perspectives of person, place, and time. Drug. Alcohol Depend. 95 (1), S74-S104.

Coupland, C., Dhiman, P., Morriss, R., Arthur, A., Barton, G., Hippisley-Cox, J., 2011. Antidepressant use and risk of adverse outcomes in older people: population based cohort study. BMJ 343, d4551.

Duffy, L., Gajree, S., Langhorne, P., Stott, D.J., Quinn, T.J., 2013. Reliability (inter-rater agreement) of the Barthel Index for assessment of stroke survivors: systematic review and meta-analysis. Stroke 44, 462-468.

Emerson, E., Hatton, C., 2007. Mental health of children and adolescents with intellectual disabilities in Britain. Br. J. Psychiatry 191, 493-499.

Farner, L., Wagle, J., Engedal, K., Flekkoy, K.M., Wyller, T.B., Fure, B., 2010. Depressive symptoms in stroke patients: a 13 month follow-up study of patients referred to a rehabilitation unit. J. Affect. Disord. 127, 211-218.

Goldstein, H., Rasbash, J., 2003. Applications and understandings of multilevel modelling in the social sciences. Economic and Social Research Council, Swindon.

Hackett, M.L., Anderson, C.S., House, A., Xia, J., 2008. Interventions for treating depression after stroke. Cochrane Database Syst. Rev., CD003437.

Hackett, M.L., Pickles, K., 2014. Part I: frequency of depression after stroke: an updated systematic review and meta-analysis of observational studies. Int. J. Stroke.

Hatano, S., 1976. Experience from a multicentre stroke register: a preliminary report. Bull. World Health Organ. 54, 541-553.

Haviland, A., Nagin, D.S., Rosenbaum, P.R., 2007. Combining propensity score matching and group-based trajectory analysis in an observational study. Psychol. Methods 12, 247-267.

Heuschmann, P.U., Grieve, A.P., Toschke, A.M., Rudd, A.G., Wolfe, C.D., 2008. Ethnic group disparities in 10-year trends in stroke incidence and vascular risk factors: the South London Stroke Register (SLSR). Stroke 39, 2204-2210.

Jones, B.L.N., D.S., 2013. A Note on a Stata Plugin for Estimating Group-based Trajectory Models. Sociol. Methods Res. (0), 1-6.

Kandel, D.B., Yamaguchi, K., Chen, K., 1992. Stages of progression in drug involvement from adolescence to adulthood: further evidence for the gateway theory. J. Stud. Alcohol 53, 447-457.

Lai, S.M., Duncan, P.W., Keighley, J., Johnson, D., 2002. Depressive symptoms and independence in BADL and IADL. J. Rehabil. Res. Dev. 39, 589-596.

Lin, H., McCulloch, C.E., Turnbull, B.W., Slate, E.H., Clark, L.C., 2000. A latent class mixed model for analysing biomarker trajectories with irregularly scheduled observations. Stat. Med. 19, 1303-1318.

Lincoln, N.B., Brinkmann, N., Cunningham, S., et al., 2013. Anxiety and depression after stroke: a 5 year follow-up. Disabil. Rehabil. 35, 140-145.

Mahoney, F.I., Barthel, D.W., 1965. Functional evaluation: the barthel index. Md. State Med .J. 14, 61-65.

McKevitt, C., Fudge, N., Redfern, J., et al., 2011. Self-reported long-term needs after stroke. Stroke 42, 1398-1403.

Meader, N., Moe-Byrne, T., Llewellyn, A., Mitchell, A.J., 2014. Screening for poststroke major depression: a meta-analysis of diagnostic validity studies. J. Neurol. Neurosurg. Psychiatry 85, 198-206.

Moffitt, T.E., Klaus-Grawe Think, T., 2013. Childhood exposure to violence and lifelong health: clinical intervention science and stress-biology research join forces. Dev. Psychopathol. 25, 1619-1634.

Muthen, B., 2006. The potential of growth mixture modelling. Infant Child. Dev. 15, $623-625$.

Muthen, B., Muthen, L.K., 2000. Integrating person-centered and variable-centered analyses: Growth mixture modeling with latent trajectory classes. Alcoholism Clin. Exp. Res. 24, 882-891.

Nagin, D., 2005. Group-Based Modeling of Development. Harvard Univ. Press, Cambridge, MA

Nagin, D.S., Odgers, C.L., 2010a. Group-based trajectory modeling (nearly) two decades later. J. Quant. Criminol. 26, 445-453.

Nagin, D.S., Odgers, C.L., 2010b. Group-based trajectory modeling in clinical research. Annu. Rev. Clin. Psychol. 6, 109-138.

NHS, N. H. S., 2015. Selective serotonin reuptake inhibitors (SSRIs). 〈http://www. nhs.uk/conditions/SSRIs-(selective-serotonin-reuptake-inhibitors)/Pages/In troduction.aspx $>$ (accessed: 01.12.15).

NICE, N. I. f. H. a. C. E., 2015. First-choice antidepressant use in adults with depression or generalised anxiety disorder. 〈https://www.nice.org.uk/advice/ ktt8>; (accessed 01.12.15).

Pearson, J.D., Morrell, C.H., Landis, P.K., Carter, H.B., Brant, L.J., 1994. Mixed-effects regression models for studying the natural history of prostate disease. Stat. Med. 13, 587-601.

Peer, J.E., Spaulding, W.D., 2007. Heterogeneity in recovery of psychosocial functioning during psychiatric rehabilitation: an exploratory study using latent growth mixture modeling. Schizophr. Res. 93, 186-193.

Rayner, L., Price, A., Evans, A., Valsraj, K., Hotopf, M., Higginson, I.J., 2011. Antidepressants for the treatment of depression in palliative care: systematic review and meta-analysis. Palliat. Med. 25, 36-51.

Robinson, R.G., 2003. Poststroke depression: prevalence, diagnosis, treatment, and disease progression. Biol. Psychiatry 54, 376-387.

StataCorp., 2013. Stata Statistical Software: Release 13. College Station, T. S. L.

Tilling, K., Sterne, J.A., Wolfe, C.D., 2001. Estimation of the incidence of stroke using a capture-recapture model including covariates. Int. J. Epidemiol. 30 (13511359), 1359-1360.

Wade, D.T., Legh-Smith, J., Hewer, R.A., 1987. Depressed mood after stroke. A community study of its frequency. Br. J. Psychiatry 151, 200-205.

White, J.H., Magin, P., Attia, J., Sturm, J., Carter, G., Pollack, M., 2012. Trajectories of psychological distress after stroke. Ann. Fam. Med. 10, 435-442.

Wolfe, C.D., Crichton, S.L., Heuschmann, P.U., et al., 2011. Estimates of outcomes up to ten years after stroke: analysis from the prospective South London Stroke Register. PloS Med. 8, e1001033.

Wolfe, C.D., Taub, N.A., Woodrow, E.J., Burney, P.G., 1991. Assessment of scales of disability and handicap for stroke patients. Stroke 22, 1242-1244.

World Health Organization, 1993. The ICD-10 classification of mental and behavioural disorders: diagnostic criteria for research. World Health Organization, Geneva.

Zigmond, A.S., Snaith, R.P., 1983. The hospital anxiety and depression scale. Acta Psychiatr. Scand. 67, 361-370.

von Elm, E., Altman, D.G., Egger, M., et al., 2007. The strengthening the reporting of observational studies in epidemiology (STROBE) statement: guidelines for reporting observational studies. Epidemiology 18, 800-804. 